\title{
Correcting the effect of refraction and dispersion of light in FT-IR spectroscopic imaging in transmission through thick infrared windows
}

\author{
K. L. Andrew Chan and Sergei G. Kazarian* \\ Department of Chemical Engineering, Imperial College London, SW7 2AZ
}

\begin{abstract}
Transmission mode is one of the most common sampling methods for FTIR spectroscopic imaging because the spectra obtained generally have a reasonable signal to noise ratio. However, dispersion and refraction of infrared light occurs when samples are sandwiched between infrared windows or placed underneath a layer of liquid. Dispersion and refraction cause infrared light to focus with different focal lengths depending on the wavelength (wavenumber) of the light. As a result, images obtained are in focus only at a particular wavenumber while they are defocused at other wavenumber values. In this work, a solution to correct this spread of focus by means of adding a lens on top of the infrared transparent window, such that a pseudo hemisphere is formed, has been investigated. Through this lens (or hemisphere), refraction of light is removed and the light across the spectral range has the same focal depth. Furthermore, the lens acts as a solid immersion objective and an increase of both magnification and spatial resolution (by 1.4 times) is demonstrated. The spatial resolution was investigated using an USAF resolution target, showing that the Rayleigh criterion can be achieved, as well as a sample with a sharp polymer interface to indicate the spatial resolution that can be expected in real samples. The reported approach was used to obtain chemical images of cross-sections of cancer tissue and hair samples sandwiched between infrared windows showing the versatility and applicability of the method. In addition to the improved spatial resolution, the results reported herein also demonstrate that the lens can reduce the effect of scattering near the edges of tissue samples. The advantages of the presented approach, obtaining FTIR spectroscopic images in transmission mode with the same focus across all wavenumber values and simultaneous improvement in spatial resolution, will have wide implications ranging from studies of live cells to sorption of drugs into tissues.
\end{abstract}

Key words: Image resolution, high spatial resolution, diffraction limits, cancer tissue, hair, Mie scattering corrections, chromatic aberration, FT-IR imaging 


\section{Introduction}

FTIR imaging has been shown to be a powerful chemical imaging method that can provide both spatial and chemical information in a single measurement. The applications of FTIR imaging are steadily expanding and include pharmaceutical materials, ${ }^{1-3}$ polymer processes, ${ }^{4-5}$ biological samples, ${ }^{6}$ forensic studies ${ }^{7}$ as well as art and heritage samples. ${ }^{8}$ FTIR imaging is mostly conducted either in transmission, reflectance or Attenuated Total Reflection (ATR) modes. These imaging modes have their own advantages and disadvantages and the choice of imaging mode is often based on the requirements of the specific application. ${ }^{9}$ One of the advantages of measuring images in ATR mode is that there is an opportunity to increase the spatial resolution of the image by using an ATR element with a hemispherical shape such as those used in infrared microscopes. ${ }^{10-12}$ In this format, the ATR element, in addition to creating the internal reflection, acts as a solid immersion lens ${ }^{13-16}$ thereby increasing the numerical aperture of the system by up to four times (e.g. when Ge is used as the ATR element) depending on the refractive index of the ATR element. Nevertheless, because of the greater pathlength, transmission mode FTIR imaging offers the advantage that the spectra obtained, generally, have a better signal to noise ratio c.f. ATR imaging. ${ }^{9}$ Transmission mode has therefore been an important approach for imaging applications, particularly in the areas of biomedical and tissue imaging. ${ }^{17-24}$ However, spatial resolution in transmission mode is lower than that of micro-ATR mode even when measured using a powerful synchrotron source. ${ }^{25-26}$ Although a recent report $^{27}$ has shown that, by using a multi-beam synchrotron source and a high magnifying power objective, the spatial resolution in transmission mode can approach that of micro-ATR mode.

When samples are measured, sandwiched between a pair of infrared transparent windows, ${ }^{28-39}$ such as in transmission liquid cells, diamond compression cells or when samples are underneath a layer of liquid, ${ }^{40}$ refraction occurs and the depth position of the focus of the infrared light becomes wavelength dependent (chromatic aberration). This effect is especially strong when a microscope with high numerical aperture objective or a thick window is used. Figure 1a illustrates the representative paraxial rays collected by a typical inverse Cassegrain objective (a type of objective that is commonly employed in infrared microspectroscopy) from the sample. The collected light is in good focus at all wavelengths because the optics employed in infrared microspectroscopy are all of reflective type, hence no refraction occurs. The working distance, $a$, is the distance from the lowest point of the objective to the sample when the sample is in focus. However, when the sample is measured through a window, as shown in Figure $1 \mathrm{~b}$, refraction occurs due to the difference in the refractive indices of the window and air and because the degree of refraction depends on the wavelength of light, dispersion of the light also occurs. As a result of the refraction and dispersion of light, the working distance is changed and as demonstrated in Figure 1c and 1d, the working distance for the longer wavelength is now different to that of the shorter wavelength. Hence, it is impossible to collect focused images across the different wavelengths of light through a thick window due to this spread of focal points. However, when a lens is used, which has been selected such that its curvature and centre thickness forms a pseudo-hemisphere when it is placed on top of the window, light does not refract when exiting the pseudo-hemisphere and hence no dispersion occurs (as shown in Figure $1 \mathrm{e})$. As a result, light at different wavelengths are all in focus at the same time.

The spread of focal points orthogonal to the sample plane is less important in single element measurement mode because the sample is usually significantly larger than the sampling area and only averaged information is collected. However, when a spatially resolved image is collected (whether using a focal plane array, a linear array detector or single point mapping with an optical aperture) this chromatic aberration will lead to blurring of images that are generated using spectral bands with wavenumber other than the one that is in focus. This effect is particularly significant for spectroscopic imaging and mapping because the range of wavelengths (or wavenumber) measured in the spectrum is usually broad. Despite 
this, the problem is hardly discussed in the literature except for a few reports where an ultra thin diamond window, which has a near-constant refractive index in the mid-IR range, was used to reduce the effect. ${ }^{41}$ It is worthy to note, that in addition to chromatic aberration, refraction at the flat $\mathrm{CaF}_{2}$ window can potentially introduce spherical aberration to the image due to differences in the focal lengths of the marginal and paraxial rays. The result of this spherical aberration, would be a "halo" near the edges of the imaged object, caused by the overlapping of the sharply focused image from the paraxial rays and the out of focus image from the marginal rays. However, significant spherical aberration through the $\mathrm{CaF}_{2}$ window is not expected because the refractive index of $\mathrm{CaF}_{2}$ window is relatively low ( 1.3-1.4) and the numerical aperture (NA) of the system is relatively small (0.4). Nevertheless, the results from the study reported in this paper could also be used to assess the significance of spherical aberration through a $\mathrm{CaF}_{2}$ window qualitatively. The issues of chromatic aberration resulting from infrared windows, causing a shift of focus, has been discussed by $\mathrm{Carr}^{25}$ and Wetzel ${ }^{42}$ for studies without the use of infrared array detectors. Carr reported that chromatic aberration cannot be easily removed or even cannot be avoided in some cases unlike spherical aberration. ${ }^{25}$

A recent study by Tobin et al. $^{43}$ reported the detrimental effect of chromatic aberration for FTIR microspectroscopic studies of live cells in liquid cells with $\mathrm{CaF}_{2}$ windows using either single element or array detectors. They showed that it was possible to reduce, but not eliminate, the effect by using thinner $\mathrm{CaF}_{2}$ windows. An alternative approach to removing chromatic aberration (and any possible spherical aberration) is to reduce or remove the refraction occuring at the air-IR window interface by use of a hemisphere. Since the infrared light is focused by the condenser and objective in the infrared microscope, the refraction of light will be removed if a hemisphere is used rather than a plain window (as illustrated in Figure 1b). However, hemispherical windows are not commonly used in infrared transmission cells, perhaps because of perceived, albeit wrongly, complication of building such device. Therefore, we have adapted a lens, made of the same material as the infrared transparent window, such that when it is placed on top of the window, the lens and the window form a pseudo hemisphere as shown in Figure 1c. In this way, the lens can be applied to existing transmission cells. This approach will not only remove the dispersion and refraction effect, the pseudo hemisphere can also act as a solid immersion lens such that the magnification and the spatial resolution would increase by the same factor as the value of refractive index of the infrared transparent material. Solid immersion lenses or oil have been used in a number of optical applications, including both reflection and transmission modes, but so far have not been applied to IR spectroscopic investigations. ${ }^{13-14,44-46}$

In this paper, the effect of chromatic aberration, caused by dispersion and refraction through infrared transparent windows for FTIR spectroscopic imaging in transmission mode, was examined. An analysis was then undertaken of FTIR images measured in transmission mode with and without the use of a pseudo hemisphere lens in order to demonstrate the removal of the chromatic aberration on the obtained images, the increased magnification and improved spatial resolution. The approach of using a pseudo-hemisphere to remove chromatic aberration was demonstrated for use with an imaging system using an inverse Cassegrain objective and for a specific infrared transmission cell with thick windows. It should be noted that this approach is expected to work well for FTIR spectroscopic imaging systems that employ Cassegrain objectives. For cases where transmissions cells of dimensions other than reported here are used the specific dimensions of the lens will need to be calculated on an individual basis." 


\section{Experimental}

FTIR images were measured using an FTIR continuous scan spectrometer (IFS 66, Bruker, Germany) attached to an infrared microscope (IRscopell, Bruker, Germany) with focal plane array detectors (64 $\times 64$ and $128 \times 128$ ). The microscope is equipped with a $15 \times$ Cassegrain objective with a numerical aperture of 0.4. Spectra with the spectral range $3800 \mathrm{~cm}^{-1}$ to $1000 \mathrm{~cm}^{-1}$ were measured with either 32 or 128 scans at 8 $\mathrm{cm}^{-1}$ spectral resolution. All spectra (4096 or 16384) were measured simultaneously and an image typically took $30 \mathrm{~s}$ to 5 minutes to measure depending on the number of pixels and the number of scans.

An infrared transparent liquid cell with a $6 \mu \mathrm{m}$ spacer (Omni cell, Specac Ltd, UK) was used. The cell consisted of two $\mathrm{CaF}_{2}$ windows, each window $4 \mathrm{~mm}$ thick, and a holder that clamped the windows together with the spacer. $\mathrm{A} \mathrm{CaF}_{2}$ lens was made with a diameter of $10 \mathrm{~mm}$, a radius of the curvature of 10 $\mathrm{mm}$ and a centre thickness of $6 \mathrm{~mm}$ (Crystran Ltd, UK) such that it formed a pseudo hemisphere when placed on a $4 \mathrm{~mm}$ thick $\mathrm{CaF}_{2}$ window.

Experiments with transmission cell without lens. A sample of human hair, $6 \mu \mathrm{m}$ cross section, and, separately, a polymer interface sample (acrylonitrile-butadiene-styrene/polyurethane) were sandwiched between the two windows with the $6 \mu \mathrm{m}$ spacer. The transmission cell was then placed on the infrared microscope stage and the light, focused on the sample, was monitored with the FPA detector. Several FTIR images were then taken at various focal lengths by changing the height of the stage incrementally.

Experiments with transmission cell with lens (pseudo hemisphere). The same samples sandwiched in the transmission cell were used. The transmission cell was placed on the infrared microscope stage with the $\mathrm{CaF}_{2}$ lens placed on top of the transmission cell window. The lens was centred on the sample such that the middle of the lens was directly above the imaged sample. The sample was focused using visible light.

Measurement of the Air Force (USAF) resolution target and microtomed tissue sample. The group 6 and group 7 elements on the USAF resolution target (Edmund Optics) have been used to estimate the resolution of the system in transmission mode. The USAF resolution target was placed on the infrared microscope and the patterns were focused using visible light. Infrared images of the sample were measured with and without the pseudo lens. The sample of tissue was collected during routine surgical biopsy and was kindly provided by Prof. Nick Stone with the appropriate ethical approval. The tissue

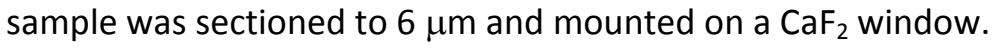

\section{Results and discussions}

The first sample investigated by this approach was a $6 \mu \mathrm{m}$ cross section of human hair. The infrared spectrum of hair has bands between $2800-3000 \mathrm{~cm}^{-1}$ (wavelength of $\sim 3.5 \mu \mathrm{m}, \mathrm{CH}$ stretching mode vibrations) and $1427-1485 \mathrm{~cm}^{-1}(\sim 7 \mu \mathrm{m}, \mathrm{CH}$ bending mode vibrations) which can be used to generate images at different wavenumber. The images in Figure $2 a$ and $b$ show that, when imaged without the lens (image a-d), if the image is focused in the $2800-3000 \mathrm{~cm}^{-1}$ region (Figure $2 \mathrm{a}$ and $\mathrm{b}$ ), then the image is out of focus in the 1427-1485 $\mathrm{cm}^{-1}$ region and vice versa. The difference in the depth of focus was found to be around $80 \mu \mathrm{m}$. A simple calculation based on Snell's law and trigonometry shows that the calculated difference in the depth of focus is around $90 \mu \mathrm{m}$ which is in good agreement with the measurements (see Supporting information a). This result clearly demonstrates that refraction at the interface between the infrared transparent window and air causes the focal length to be wavelength (or wavenumber) 
dependent. However, when a lens is placed on top of the window to create a pseudo hemisphere of $\mathrm{CaF}_{2}$, light does not refract and the images remain in focus at both regions, $2800-3000 \mathrm{~cm}^{-1}$ and $1427-1485 \mathrm{~cm}^{-1}$ (see Figure $2 \mathrm{e}$ and $\mathrm{f}$ ). In fact, the focal length is the same in visible light and infrared light when the lens is used removing the need to check that the image is in focus using the FPA detector.

The images obtained with the lens also show an increase in magnification and, as a result, the centre of the hair where the medulla is located is more clearly visible (Figure 2a and e). Spectra were extracted from the medulla region of the hair and compared to the spectrum extracted from the same area when the lens was not used (Figure 3). (Note that for the measurement without the lens, the spectrum was extracted from the image where the infrared light was focused in the region 1427-1485 $\mathrm{cm}^{-1}$.) The spectrum extracted from the image without the lens does not clearly show the characteristic $\mathrm{COO}^{-}$band of the medulla ${ }^{47}$ $\left(\sim 1580 \mathrm{~cm}^{-1}\right)$ despite the focus being optimised near this wavenumber. However, the spectrum extracted from the image measured with the lens clearly shows the characteristic $\mathrm{COO}^{-}$band at $1580 \mathrm{~cm}^{-1}$, suggesting that the spatial resolution of the image measured with the lens is better than when measured without lens. From the size of the hair, one can also estimate that the magnification through the pseudo hemisphere is approximately 1.4 times. In terms of spherical aberration, there is no significant "halo" near the edges of the hair demonstrating that spherical aberration is not significant in the images collected either with or without the lens.

For a more clear comparison of spatial resolution and imaging size, the USAF spatial resolution target was measured with and without the pseudo hemisphere. Images were generated by plotting the transmittances at each pixel at $3100 \mathrm{~cm}^{-1}(\lambda \sim 3.2 \mu \mathrm{m})$ (Figure 4). In this case, the USAF target was used to measure the imaging area and spatial resolution rather than using the imaging system to measure the spacing of the USAF target. The image measured without the pseudo hemisphere (Figure 4a) clearly has a lower magnification and spatial resolution than the image measured with the pseudo hemisphere (Figure 4b). The transmittance profiles, extracted along the lines indicated on the images, are shown in Figure $4 c$ and $d$. The spacing between the stripes in the pattern is stated below the profile. The parallel dotted lines indicate the minimum contrast of $26.4 \%$ that is required to satisfy the Rayleigh criterion. From this analysis, the profile indicates that in Figure 4 a features of $7 \mu \mathrm{m}$ cannot be resolved. However, one group of stripes (indicated by the arrow) with $4.9 \mu \mathrm{m}$ separation was resolved. Considering that the wavelength of light used to generate this image was $3.2 \mu \mathrm{m}$ and the numerical aperture of this objective was 0.4, the calculated Rayleigh criterion is $4.9 \mu \mathrm{m}$, suggesting that the smallest feature that can be resolved in this image should be the pattern with $4.9 \mu \mathrm{m}$ separation. Previous work on transmission FTIR imaging has shown that to achieve diffraction limited spatial resolution, over sampling of the imaging acquisition is required. ${ }^{27}$ The size of the imaging area in Figure $4 \mathrm{a}$ is $341 \mu \mathrm{m} \times 341 \mu \mathrm{m}$ with $128 \times 128$ pixels measured, giving a projected pixel size of $2.67 \mu \mathrm{m}$, around 1.8 times oversampling at $3100 \mathrm{~cm}^{-1}(3.2 \mu \mathrm{m})$. Apparently even greater oversampling would be required to consistently obtain the expected spatial resolution, which is in good agreement with the previous work. ${ }^{27}$ In comparison, the profile extracted from the image measured with the pseudo hemisphere shows that the smallest resolvable feature is $3.5 \mu \mathrm{m}$. According to the Rayleigh criterion, the smallest feature expected to be resolved with the hemisphere is $3.5 \mu \mathrm{m}$ which is in good agreement with the result found here.

The increase in magnification was expected to be the same as the refractive index of $\mathrm{CaF}_{2}$ which is 1.4. The increase in magnification can be measured by comparing the group 7 transmittance profiles extracted from Figure $4 \mathrm{a}$ and $4 \mathrm{~b}$. The whole section of the group 7 transmittance profile extracted from Figure $4 \mathrm{a}$ spans across 45 pixels while the one extracted from Figure $4 \mathrm{~b}$ spans across 64 pixels, therefore the 
increase in magnification is measured to be 1.42 times, which is again in good agreement with the expected value. The size of the imaging area for Figure $4 \mathrm{~b}$ is therefore $240 \mu \mathrm{m}$ and the projected pixel size is $1.88 \mu \mathrm{m}(128 \times 128$ pixels $)$.

The resolution test using the USAF resolution target may be used as a tool to compare the attainable spatial resolution in imaging systems. However, the resolution USAF target method, while often being used as an indicator of spatial resolution based on the Rayleigh criterion, cannot be used to predict the expected spatial resolution, which is the minimum distance needed to extract spectrum with insignificant spectral contribution from another neighboring material. Apart from the difference in criteria, real samples are usually significantly thicker than the metal film on the USAF resolution target and usually with much smaller refractive index contrast than the target. Therefore the expected spatial resolution of a sample that was more representative of a real sample was investigated. Therefore the expected spatial resolution of a sample that was more representative of a real sample was investigated. A $6 \mu \mathrm{m}$ thick laminate of polymer films, with a sharp interface, was measured to demonstrate the expected spatial resolution. The results of these measurements are shown in Figure 5 . The polymer on the left has a characteristic spectral band at $3130 \mathrm{~cm}^{-1}$ (wavelength of $3.2 \mu \mathrm{m}$ ) while the polymer on the right has a spectral band at $3030 \mathrm{~cm}^{-1}$ (wavelength of $3.3 \mu \mathrm{m}$ ) that can be used to generate images representing the spatial distribution of the polymers. These bands were chosen because they have similar wavelengths to the one used in the spatial resolution analysis with the USAF resolution target in Figure 4, allowing a direct comparison to be made between the two sets of results. The images measured with and without the lens through the transmission cell appear to have similar sharpness. The focus also appears to be good across the whole length of the interface without significant "halo" near the edges demonstrating that there is no significant spherical aberration in images collected with or without the lens. However, the acquired images have areas of $170 \mu \mathrm{m} \times 170 \mu \mathrm{m}$ when no lens is used and $120 \mu \mathrm{m} \times 120 \mu \mathrm{m}$ when the pseudo hemisphere is used (for a $64 \times 64$ pixels FPA). The increase in magnification without any worsening of the sharpness of the image is an indication that the spatial resolution has also increased. Absorbance profiles across the interfaces have been extracted and are shown in the bottom row in Figure 5. Spectra measure with the lens and extracted across the profiles in Figure 5 are shown in Supporting information b. It is clear from the results that it requires approximately 6 pixels for the profiles to drop across the interface. Multiplying this to the projected pixel size, the spatial resolution is $16 \mu \mathrm{m}$ for the images obtained without the lens (which is consistent with the spatial resolution obtained by us earlier ${ }^{26}$ ) and $11 \mu \mathrm{m}$ for the images measured with the lens. This is approximately 3 times greater than the values obtained when the USAF resolution target was used. This is significant because the resolution test performed by measuring the absorbance profile across the interface of the polymer laminate film provides the minimal distance that is required for a spectrum to be free of spectral "contamination" from its surrounding pixels. This information is particularly important because real samples often present mixtures of different components (e.g. biological samples). This result points to the fact that while the USAF target may provide the minimum distance that is required to resolve nearby objects, ${ }^{27}$ the actual spatial resolution that is important for spectroscopic imaging of real samples (e.g. the $6 \mu \mathrm{m}$ thick film used in this experiment), is actually about 3 times worse.

Having established the increase in spatial resolution using the pseudo hemisphere, its application to samples measured in transmission mode when a liquid cell is not used was also investigated. A $10 \mu \mathrm{m}$ thick breast cancer tissue cross section was used. The tissue was placed on the top surface of a $\mathrm{CaF}_{2}$ window . A measurement was taken and the result is shown in Figure 6a. The general features of the tissue are clearly 
visible with the image generated using the amide I band $\left(1720-1596 \mathrm{~cm}^{-1}\right)$. The sample was then imaged in the same area with the pseudo hemisphere rested on top of the tissue. Since no pressure was applied, the tissue was not damaged by the pseudo hemisphere with the tissue remaining intact after the measurement. The image measured with the lens, which is shown in Figure $6 \mathrm{~b}$, is clearly sharper. Although the gain in spatial resolution is relatively small, the effort to apply the lens is minimal and can be readily applied to various samples to achieve higher spatial resolution images. Multi-beam synchrotron FTIR instruments with an FPA detector can be used to achieve higher spatial resolution c.f. "standard" transmission mode, due to the high brightness of the source enabling the use of an optical objective with higher magnifying power and numerical aperture (NA of 0.65 as compared to NA of 0.4 , hence an improvement of $60 \%)$. That approach has already been shown to improve the spatial resolution when applied to the imaging of tissue cross sections. ${ }^{48}$ The hemisphere lens (or the pseudo hemisphere) presented here would be readily adaptable in such a system to further increase the spatial resolution by 40 $\%$.

When applying the method reported herein to other FTIR imaging setups, consideration must be given to the refractive index of the material used. Although higher magnification and spatial resolution may be achieved when using material of higher refractive index, (for example, ZnSe has a refractive index of 2.4 which could potentially increase the spatial resolution by 2.4 times) if the refractive index is too great (e.g. Ge has a refractive index of 4 and Si has a refractive index of $\sim 3.1$ ), the infrared light will start to undergo total internal reflection and become an ATR imaging system rather than a transmission imaging system.

Further analysis of the breast cancer tissue data showed that the lens also reduced some optical artefacts that are generated near the edge of the tissue due to scattering effects. Spectra were extracted across the edge of one of the two larger holes on the left hand side of the image measured with and without the lens (indicated by a straight line) and are shown in bottom of Figure 6 . When the spectra were extracted near the edges of the hole, without the lens, a typical derivative type base line near the amide I band was observed due to optical scattering. ${ }^{49-51}$ However, the spectra extracted from the measurements with the pseudo lens show that they were less affected by this scattering. The lens apparently reduces the scattering of light from regions of the sample where the considerable irregularities are present. The marked changes in the baseline below $1000 \mathrm{~cm}^{-1}$ is associated with the transmission cut-off threshold of the $\mathrm{CaF}_{2}$ windows and the lens. It should also be noted that the signal to noise ratio seems to be slightly lower when the image is measured under the pseudo lens. This can be understood by considering the fact that only one pseudo lens was used in this experiment. As a result, the amount of light collected by the detector was reduced because in this arrangement the condenser side of the optics does not match with the objective side of the optics, so a rather large portion of the light focused by the condenser will not be captured by the objective and therefore the signal to noise is somewhat lower than without the lens. However, this could be corrected by placing another lens under the transmission cell to improve the collection of light. A more detailed study of the reduction of the scattering effect, an exploration of the opportunity to measure samples that scatter in transmission mode and the improvement on the throughput of light by use of another set of lenses will be conducted and published elsewhere in due course. 


\section{Conclusions}

The focal length of IR light is strongly affected by dispersion and refraction, when transmission FTIR imaging is performed through a standard transmission infrared liquid cell, which has been demonstrated herein. The focus of light was observed to be wavelength dependent such that one can focus the image at one wavenumber while the image remains out of focus at other wavenumber. We have demonstrated that by introducing a lens on top of the window of a standard transmission infrared liquid cell, a pseudo hemisphere lens is formed on the sample and the dispersion and refraction effects are removed. The lens also acts as a solid immersion lens which increases the magnification of the system as well as the spatial resolution of the image. The spatial resolution measured with the USAF resolution target has shown that the Rayleigh criterion is reached using the lens with a resolution of $3.5 \mu \mathrm{m}$. However, when the spatial resolution is measured in transmission mode using a sharp polymer laminate interface cross section (to represent a real sample), the absorbance step function profile took $11 \mu \mathrm{m}$ to complete (at the wavenumber ca. $3000 \mathrm{~cm}^{-1}$ ) illustrating that for real samples it would take $11 \mu \mathrm{m}$ to ensure that spectra are free from spectral "contamination" from nearby pixels which is approximately 3 time worse than the Rayleigh criterion. This pseudo lens was also found to offer improvements to the measurement of samples in transmission mode without the need for contact with the sample even when a liquid cell was not used. Scattering effects across the edge of the tissue were also minimised by the presence of the lens. The benefit of the gain in spatial resolution and the reduction in spectral artefacts are therefore readily applicable to any spectroscopic imaging measurement in transmission mode, as was demonstrated here on examples of cross-sections of tissue and hair. This is the first time, to the best of our knowledge, that this approach of removing both chromatic aberration and increasing spatial resolution has been reported for FTIR spectroscopic imaging in transmission mode.

The demonstrated advantages of the presented approach, obtaining FTIR spectroscopic images in transmission mode with the same focus across all wavenumber values and simultaneous improvement in spatial resolution, will have wide implications ranging from studies of live cells to sorption of drugs into tissues. Therefore, the approach presented here not only solves the issues associated with light refraction through infrared windows for studying many samples (polymeric materials and biological tissues) but also provides exciting opportunities for dynamic studies using FTIR imaging in transmission mode, such as sorption of substances (e.g. drug molecules) into samples of any biological tissue sandwiched between two widows with the chemical images obtained focused at all wavenumber. Furthermore, this approach would be particularly useful for studies of live cells with FTIR imaging in transmission with ${ }^{43}$ or without the use of a synchrotron source. This presents new possibilities for the application of FTIR imaging in transmission mode to living biological systems. The ease of adaptation of the described approach to FTIR microscopes also provides an exciting opportunity for this to be used with the multi-beam synchrotron FTIR imaging to further increase the spatial resolution.

\section{Acknowledgements}

SGK acknowledges research funding from the European Research Council under the European Community's Seventh Framework Programme (FP7/2007-2013)/ERC advanced grant agreement no. [227950]. We also thank Neil Everall and Nick Stone for samples.

\section{References}

1. J. A. Kimber, S. G. Kazarian, F. Stepanek, Chem. Eng. Sci. 2012, 69. 394-403.

2. P. S. Wray, G. S. Clarke, S. G. Kazarian, J. Pharm. Sci. 2012, 100. 4745-4755. 
3. B. Van Eerdenbrugh, L. S. Taylor, Int. J. Pharm. 2011, 417. 3-16.

4. O. S. Fleming, K. L. A. Chan, S. G. Kazarian, Polymer 2006, 47. 4649-4658.

5. J. L. Koenig, J. P. Bobiak, Macromol. Mater. Eng. 2007, 292. 801-816.

6. S. G. Kazarian, K. L. A. Chan, Biochim. Biophys. Acta.-Biomem. 2006, 1758. 858-867.

7. C. Ricci, S. G. Kazarian, Surf. Interface Anal. 2010, 42. 386-392.

8. E. Joseph, C. Ricci, S. G. Kazarian, R. Mazzeo, S. Prati, M. loele, Vib. Spectrosc. 2010, 53. 274-278.

9. S. G. Kazarian, K. L. A. Chan, 2006, Progress in Colloid and Polymer Science,132. 1-6.

10. A. J. Sommer, L. G. Tisinger, C. Marcott, G. M. Story, 2001, 55. 252-256.

11. K. L. A. Chan, S. G. Kazarian, Appl. Spectrosc. 2003, 57. 381-389.

12. S. G. Kazarian, K. L. A. Chan, Appl. Spectrosc. 2010, 64. 135A-152A.

13. Q. Wu, L. P. Ghislain, V. B. Elings, Proc. IEEE 2000, 88. 1491-1498.

14. D. A. Fletcher, K. B. Crozier, C. F. Quate, G. S. Kino, K. E. Goodson, D. Simanovskii, D. V. Palanker, Appl. Phys. Lett. 2000, 77. 2109-2111.

15. S. B. Ippolito, B. B. Goldberg, M. S. Ünlü, Appl. Phys. Lett. 2001, 78. 4071-4073.

16. K. A. Serrels, E. Ramsay, P. A. Dalgarno, B. D. Gerardot, J. A. O'Connor, R. H. Hadfield, R. J.

Warburton, D. T. Reid, J. Nanophotonics 2008, 2. 021854.

17. E. P. Paschalis, K. Verdelis, S. B. Doty, A. L. Boskey, R. Mendelsohn, M. Yamauchi, J. Bone and Mineral Research 2001, 16. 1821-1828.

18. M. Diem, M. Romeo, S. Boydston-White, M. Miljkovic, C. Matthaus, Analyst 2004, 129. 880-885.

19. C. Krafft, L. Shapoval, S. B. Sobottka, K. D. Geiger, G. Schackert, R. Salzer, Biochim. Biophys. ActaBiomembr. 2006, 1758. 883-891.

20. R. Bhargava, Anal. Bioanal. Chem. 2007, 389. 1155-1169.

21. B. Bird, K. Bedrossian, N. Laver, M. Miljkovic, M. J. Romeo, M. Diem, Analyst 2009, 134. 1067-1076.

22. E. Giorgini, C. Conti, P. Ferraris, S. Sabbatini, G. Tosi, C. Rubini, L. Vaccari, G. Gioacchini, O.

Carnevali, Anal. Bioanal. Chem. 2010, 398. 3063-3072.

23. C. Krafft, M. A. Diderhoshan, P. Recknagel, M. Miljkovic, M. Bauer, J. Popp, Vib. Spectrosc. 2011, 55. 90-100.

24. B. Bird, M. Miljkovic, N. Laver, M. Diem, Technol. Cancer Res. Treat. 2011, 10. 135-144.

25. G. L. Carr, Rev. Sci. Instrum. 2001, 72. 1613-1619.

26. K. L. A. Chan, S. G. Kazarian, A. Mavraki, D. R. Williams, Appl. Spectrosc. 2005, 59. 149-155.

27. M. J. Nasse, M. J. Walsh, E. C. Mattson, R. Reininger, A. Kajdacsy-Balla, V. Macias, R. Bhargava, C. J. Hirschmugl, Nat. Methods 2011, 8. 413-U58.

28. C. M. Snively, J. L. Koenig, 1998, 31. 3753-3755.

29. C. M. Snively, J. L. Koenig, J. Polym. Sci. B: Polym. Phys. 1999, 37. 2261-2268.

30. T. Ribar, J. L. Koenig, R. Bhargava, 2001, 34. 8340-8346.

31. J. L. Koenig, Adv. Mat. 2002, 14. 457-460.

32. J. Gonzalez-Benito, J. L. Koenig, Macromolecules 2002, 35. 7361-7367.

33. D. W. Rafferty, J. L. Koenig, J. Contr. Rel. 2002, 83. 29-39.

34. E.-M. Shin, T. Ribar, J. L. Koenig, N. Wright, Appl. Spectrosc. 2001, 55. 1573-1579.

35. J. P. Bobiak, J. L. Koenig, J. Control. Release 2005, 106. 329-338.

36. M. S. Lindblad, B. M. Keyes, L. M. Gedvilas, T. G. Rials, S. S. Kelley, Cellulose 2008, 15. 23-33.

37. A. Gupper, K. L. A. Chan, S. G. Kazarian, Macromolecules 2004, 37. 6498-6503.

38. A. Gupper, S. G. Kazarian, Macromolecules 2005, 38. 2327-2332.

39. K. L. A. Chan, F. H. Tay, C. Taylor, S. G. Kazarian, Appl. Spectrosc. 2008, 62. 1041-1044.

40. H. Y. N. Holman, H. A. Bechtel, Z. Hao, M. C. Martin, Anal. Chem. 2010, 82. 8757-8765.

41. M. J. Nasse, S. Ratti, M. Giordano, C. J. Hirschmugl, Appl. Spectrosc. 2009, 63. 1181-1186.

42. D. L. Wetzel, Vib. Spectrosc. 2002, 29. 291-297.

43. M. J. Tobin, L. Puskar, R. L. Barber, E. C. Harvey, P. Heraud, B. R. Wood, K. R. Bambery, C. T. Dillon, K. L. Munro, Vib. Spectrosc. 2010, 53. 34-38.

44. S. Kim, H. Murakami, M. Tonouchi, IEEE J. Sel. Top. Quantum Electron. 2008, 14. 498-504.

45. N. J. Everall, Appl. Spectrosc. 2009, 63. 245A-262A.

46. D. A. Woods, C. D. Bain, Analyst 2012, 137. 35-48. 
47. G. J. Zhang, L. Senak, D. J. Moore, J. Biomed. Opt. 2011, 16. 7.

48. M. Z. Kastyak-Ibrahim, M. J. Nasse, M. Rak, C. Hirschmugl, M. R. Del Bigio, B. C. Albensi, K. M. Gough, Neuroimage 2012, 60. 376-383.

49. A. J. Hobro, B. Lendl, Vib. Spectrosc. 2011, 57. 213-219.

50. K. R. Bambery, B. R. Wood, D. McNaughton, Analyst 2012, 137. 126-132.

51. P. Bassan, A. Sachdeva, A. Kohler, C. Hughes, A. Henderson, J. Boyle, J. H. Shanks, M. Brown, N. W. Clarke, P. Gardner, Analyst 2012, 137. 1370-1377.

52. M. A. Malone, S. Prakash, J. M. Heer, L. D. Corwin, K. E. Cilwa, J. V. Coe, J. Chem. Phys. 2010, 133. 185101. 
(a)

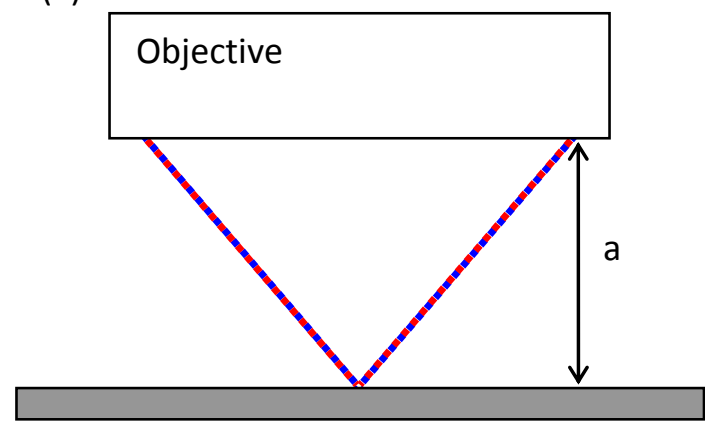

(c)

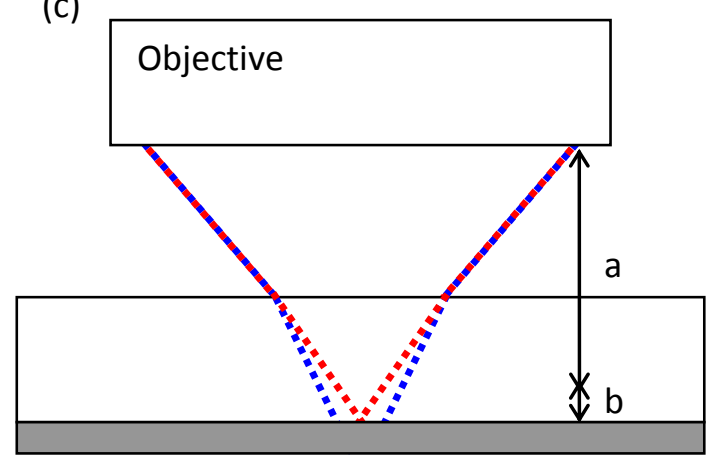

(e)

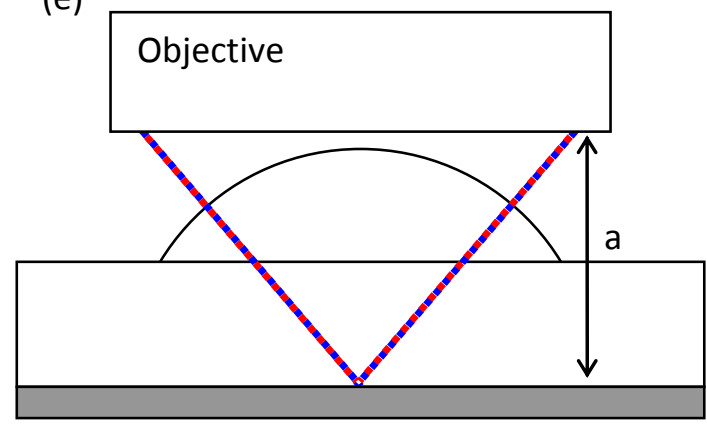

(b)

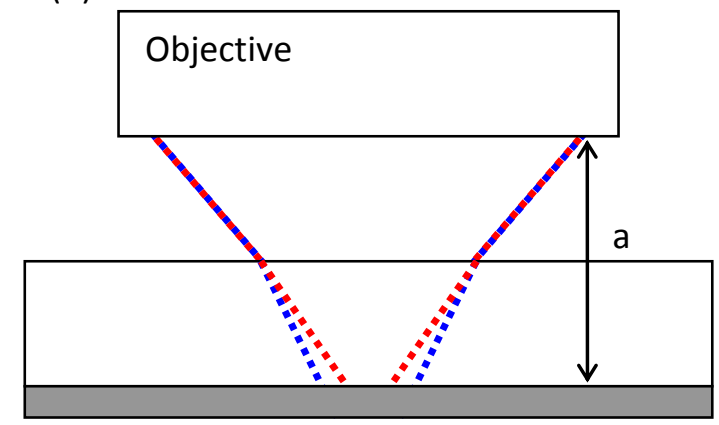

(d)

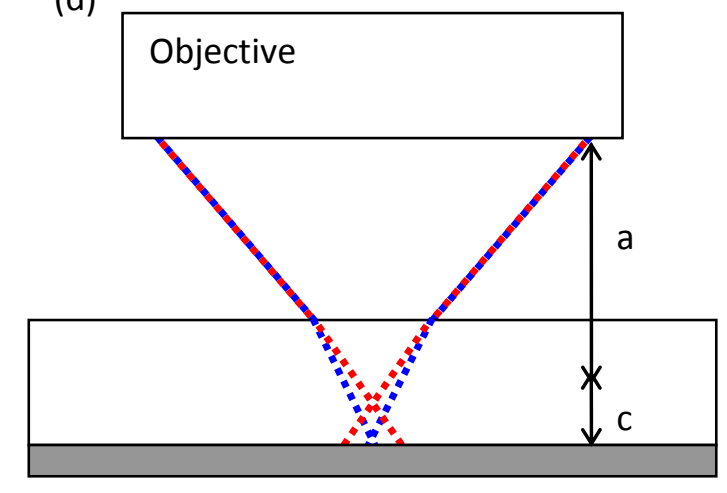

Figure 1: Schematic diagrams showing transmission imaging a) with the sample in focus. All wavelengths are in focus because the objective employs all reflective optics; b)through a thick window without adjusting the microscope stage height; c)through a thick window with adjustment of the microscope stage height to focus the longer wavelength of light (the red dotted line); d) through a thick window with adjustment of the microscope stage height to focus the shorter wavelength of light (the blue dotted line); e) through a pseudo hemisphere that is constructed by placing a lens on top of the flat infrared transparent window such that the light does not refract and all wavelengths remain in focus(red and blue dotted lines). 


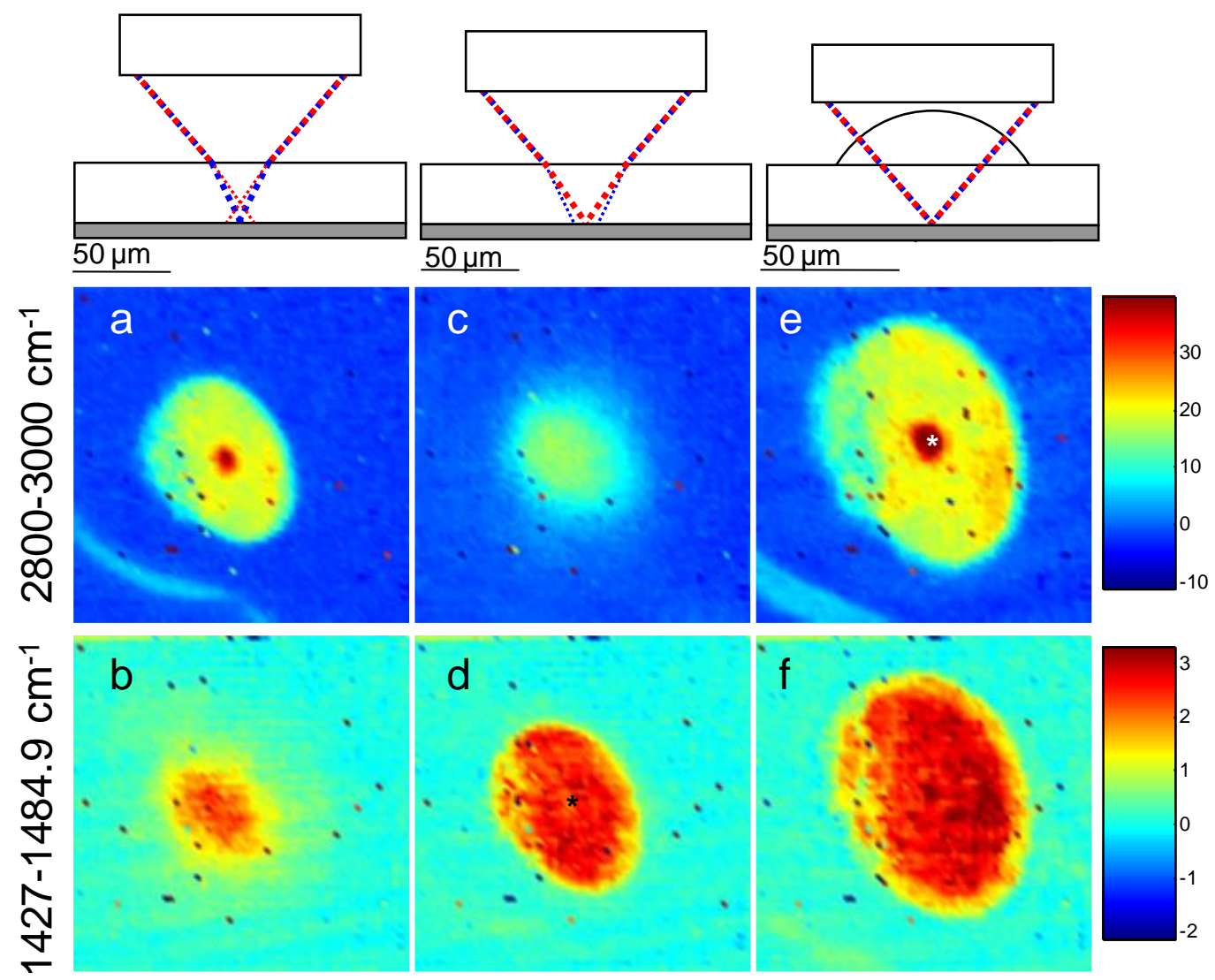

Figure 2:

Transmission FTIR imaging of a cross section of human hair a) generated using the bands between 2800 $\mathrm{cm}^{-1}$ and $3000 \mathrm{~cm}^{-1}$, measured without lens and focused at $2800-3000 \mathrm{~cm}^{-1}$ region; b) generated using the bands between $1427 \mathrm{~cm}^{-1}$ and $1485 \mathrm{~cm}^{-1}$, measured without lens and focused at 2800-3000 $\mathrm{cm}^{-1}$ region; c) generated using the bands between $2800 \mathrm{~cm}^{-1}$ and $3000 \mathrm{~cm}^{-1}$, measured without lens and focused at $1427-$ $1485 \mathrm{~cm}^{-1}$ region; d) generated using the bands between $1427 \mathrm{~cm}^{-1}$ and $1485 \mathrm{~cm}^{-1}$, measured without lens and focused at 1427-1485 $\mathrm{cm}^{-1}$ region; e) generated using the bands between $2800 \mathrm{~cm}^{-1}$ and $3000 \mathrm{~cm}^{-1}$ and measured lens; f) generated using the bands between $1427 \mathrm{~cm}^{-1}$ and $1485 \mathrm{~cm}^{-1}$ and measured lens. Image a-d has an image size of $170 \mu \mathrm{m} \times 170 \mu \mathrm{m}$ while image e and f have a image size of $120 \mu \mathrm{m} \times 120 \mu \mathrm{m}$. 


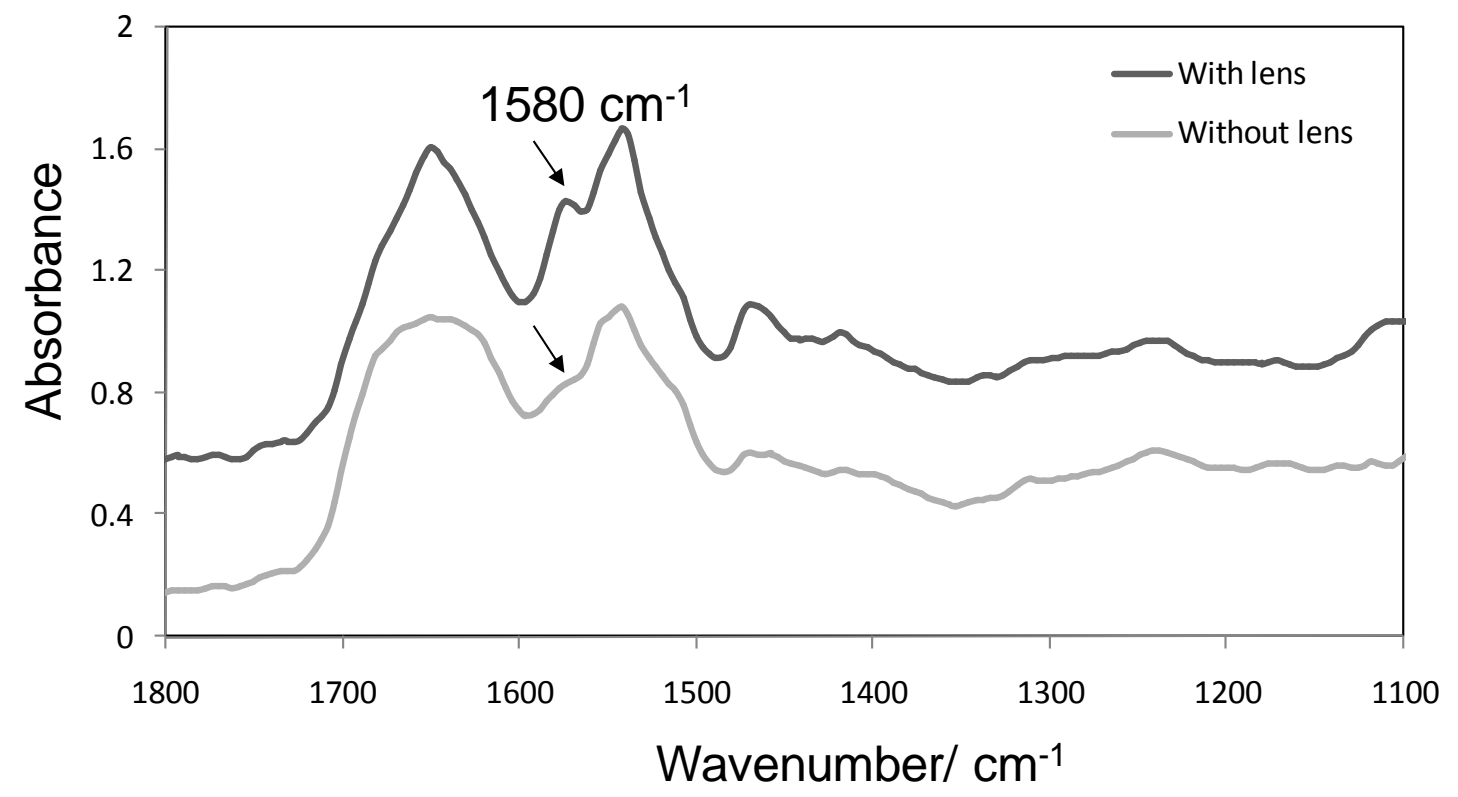

Figure 3: Extracted spectra from the medulla region (marked with a "*”) in Figure $2 d$ (pink line) and Figure 2e (blue line). 

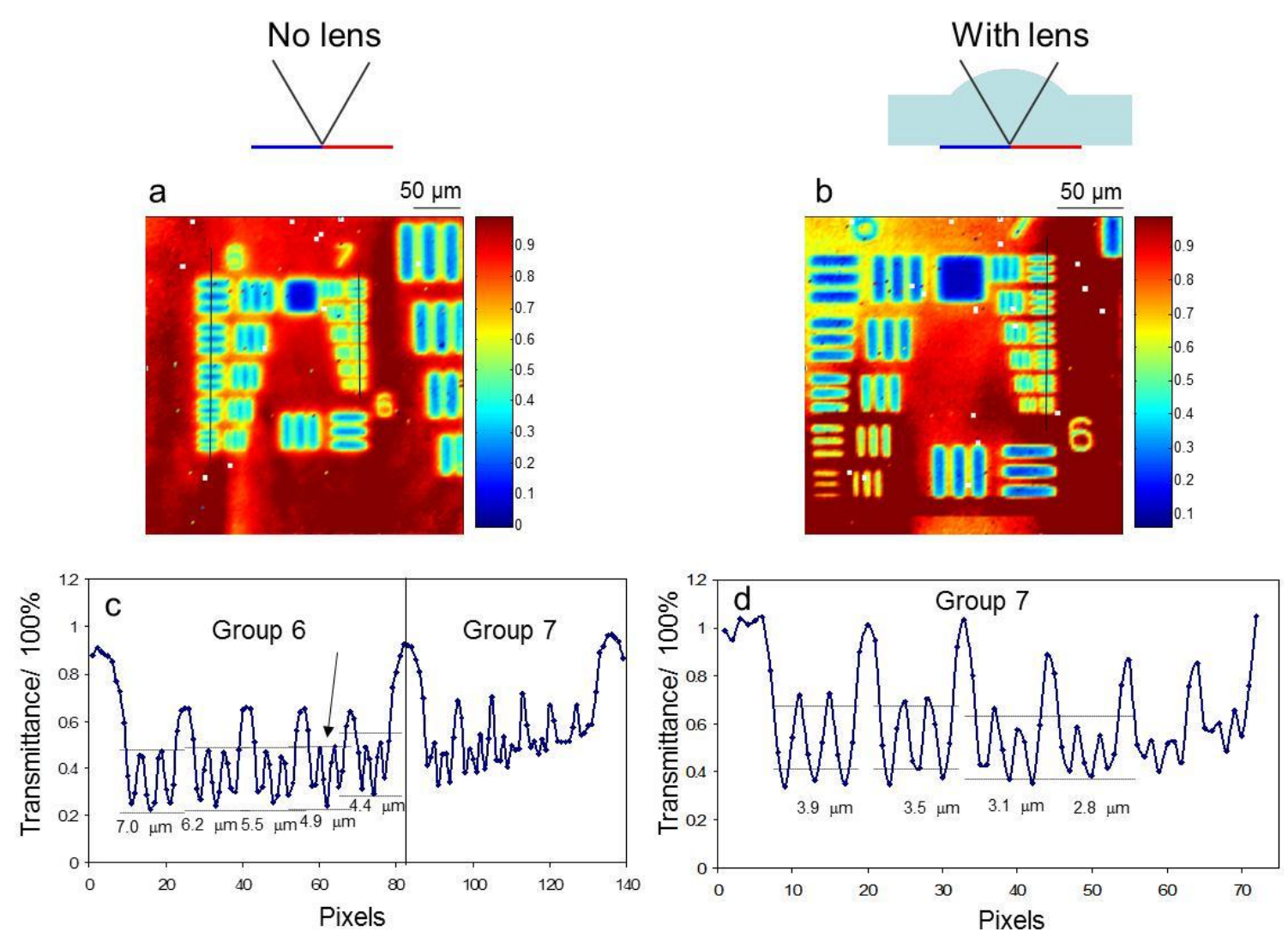

Figure 4: Transmission FTIR images of the USAF spatial resolution target a) without lens (imaging size of $341 \mu \mathrm{m} \times 341 \mu \mathrm{m}$ ) and b) with lens (imaging size of $240 \mu \mathrm{m} \times 240 \mu \mathrm{m}$ ). Images are generated by plotting the transmittance at $3100 \mathrm{~cm}^{-1}$ across the imaged area. c) Extracted transmittance profiles along the group 6 and group 7 elements of the resolution target (along the two vertical lines shown in the image a). d) Extracted transmittance profiles along the group 7 elements of the resolution target (along the vertical lines shown in the image b). 

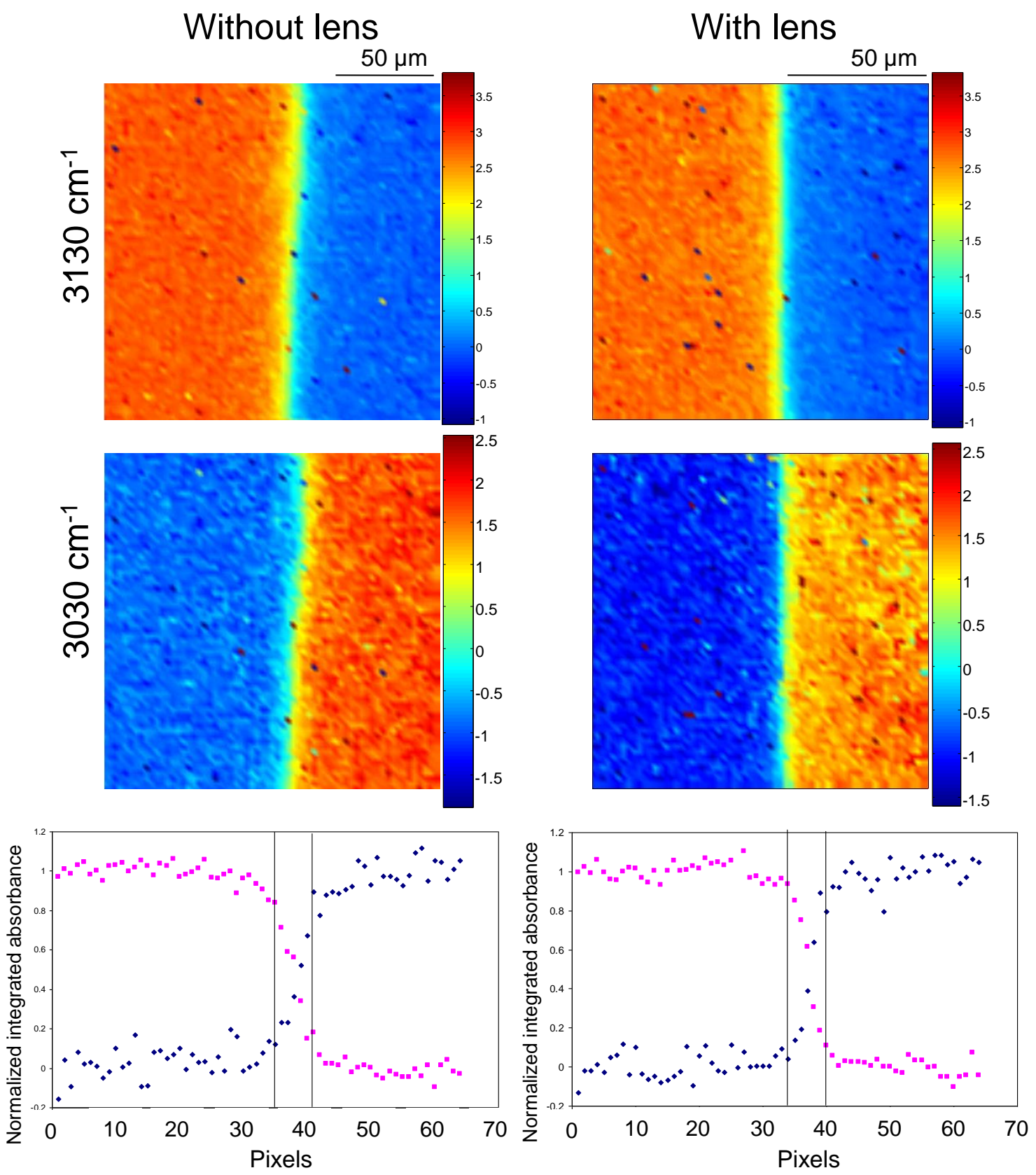

Figure 5: Transmission FTIR images of the polymer interface measured without lens (left column) and with lens (right column). Images are generated using the band at $3130 \mathrm{~cm}^{-1}$ (top row) and $3030 \mathrm{~cm}^{-1}$ (bottom row). Extracted absorbance profiles across the interface are shown at the bottom of each column. Blue diamond represents the profile of the polymer absorbance on the right hand side of the image, pink square represents the profile of the polymer absorbance on the left hand side of the image. Imaging without lens has an image size of $170 \mu \mathrm{m} \times 170 \mu \mathrm{m}$ while imaging with have an image size of $120 \mu \mathrm{m} \times 120$ $\mu \mathrm{m}$. 

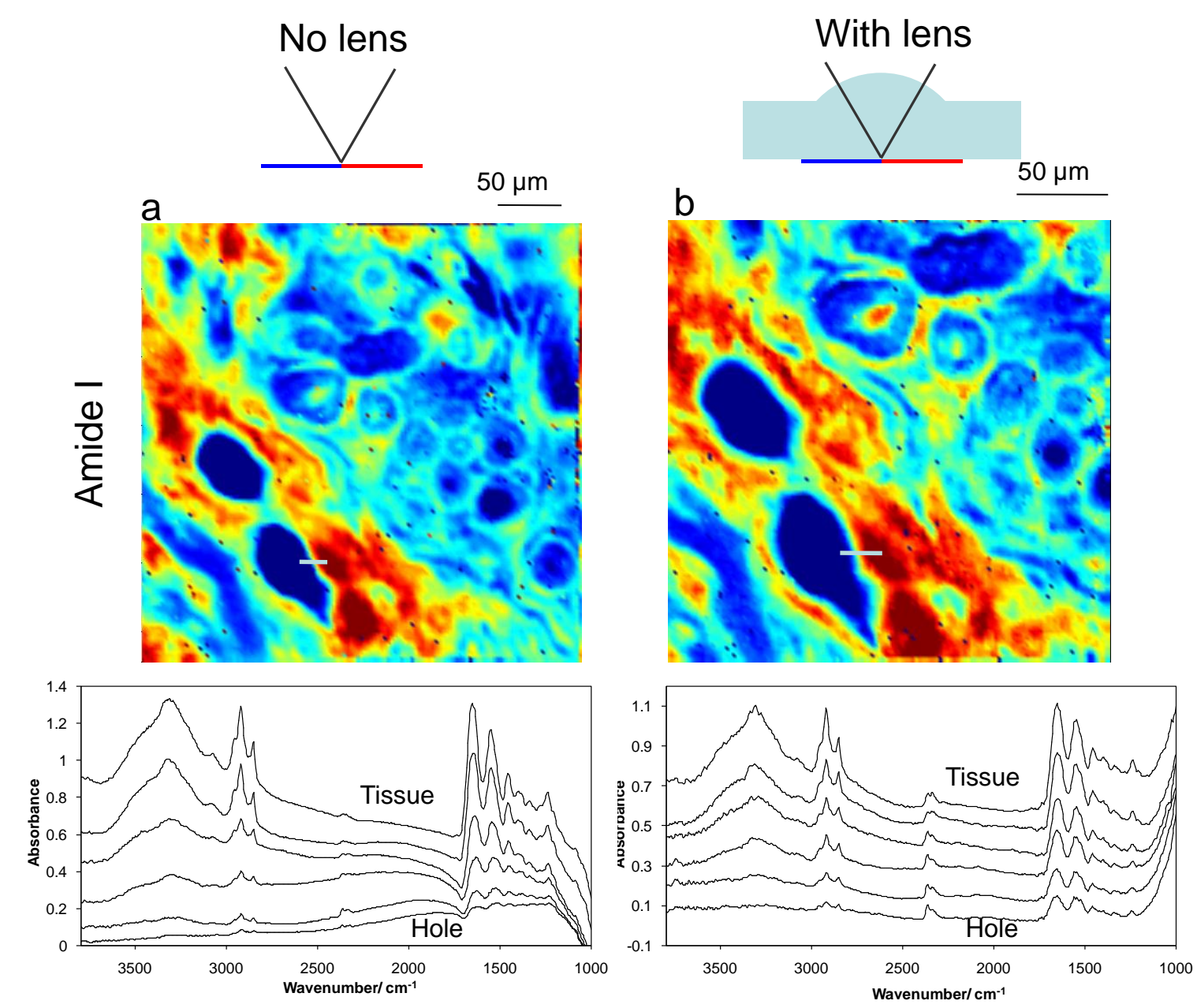

Figure 6: Transmission FTIR images of a breast tissue cross section measured (a) without lens (left ) and (b) with lens (right). Images are generated using the amide I band $\left(1720-1596 \mathrm{~cm}^{-1}\right)$. Spectra extracted from the images of the tissue along the white lines are shown at the bottom of the image. The image generated without the lens has a size of $341 \mu \mathrm{m} \times 341 \mu \mathrm{m}$ while the image measured with the lens has a size of 240 $\mu \mathrm{m} \times 240 \mu \mathrm{m}$. 
for TOC only:

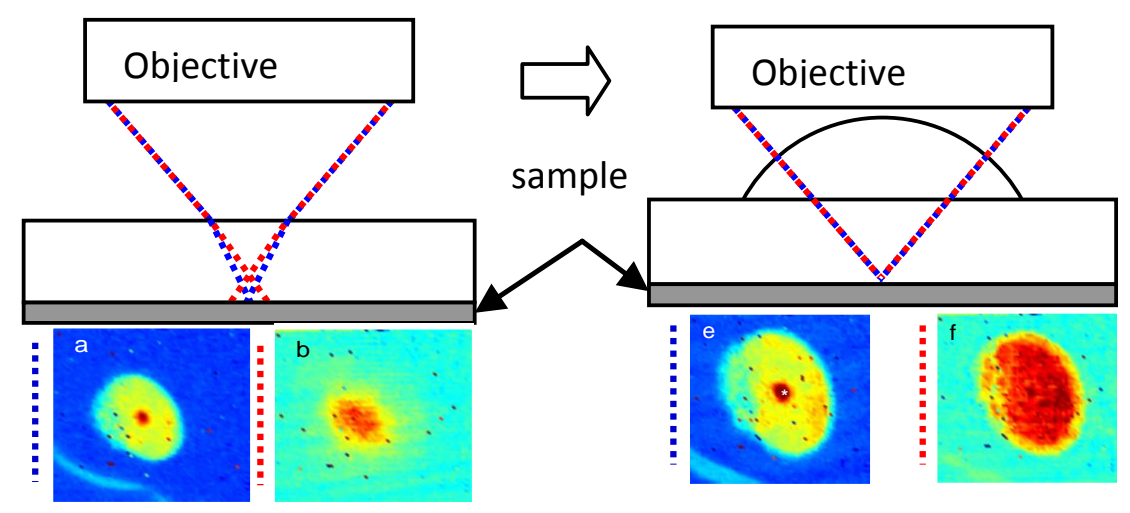

